\title{
Secant and Cosecant Sums and Bernoulli-Nörlund Polynomials
}

\author{
Peter Grabner and Helmut Prodinger
}

Institut für Analysis und Computational Number Theory (Math A) 
Quaestiones Mathematicæ 30 (2007), 159-165

\title{
SECANT AND COSECANT SUMS AND BERNOULLI-NÖRLUND POLYNOMIALS
}

\author{
PETER J. GRABNER ${ }^{\dagger}$ AND HELMUT PRODINGER ${ }^{*}$
}

ABStract. We give explicit formulæ for sums of even powers of secant and cosecant values in terms of Bernoulli numbers and central factorial numbers.

\section{INTRODUCTION}

We derive explicit formulæ for the secant sum

$$
S_{2 m}(N):=\sum_{k=1}^{\left\lfloor\frac{N-1}{2}\right\rfloor} \frac{1}{\cos ^{2 m} \frac{k \pi}{N}}
$$

and the cosecant sum

$$
C_{2 m}(N):=\sum_{k=1}^{\left\lfloor\frac{N-1}{2}\right\rfloor} \frac{1}{\sin ^{2 m} \frac{k \pi}{N}} .
$$

This research is inspired by the paper [2], where such formulæ were given for $m \leq 6$. Our approach, which uses contour integrals and residues, produces such formulæ quite effortlessly for any $m$. The main contribution of the present paper is the identification of the occurring coefficients as "classical" combinatorial quantities such as central factorial numbers and Bernoulli numbers.

\section{CONTOUR INTEGRALS AND RESIDUES}

We consider the secant sum first and start with the contour integral

$$
\frac{1}{2 \pi i} \oint_{R_{T}} \frac{1}{\cos ^{2 m} \pi z} \pi N \cot (\pi N z) d z
$$

where $R_{T}$ is the rectangle with corners $-\frac{1}{2 N} \pm i T, 1-\frac{1}{2 N} \pm i T$. By periodicity of the integrand, the integrals along the vertical lines cancel. Furthermore, the integrals along the horizontal lines tend to 0 when $T \rightarrow \infty$, since cot remains bounded and cos tends to infinity exponentially.

\footnotetext{
$\dagger$ This author is supported by the Austrian Science Foundation FWF, project S9605, part of the Austrian National Research Network "Analytic Combinatorics and Probabilistic Number Theory".

* This author is supported by the grant NRF 2053748 of the South African National Research Foundation.
} 
Thus we have

$$
\begin{aligned}
0=\frac{1}{2 \pi i} \oint_{R_{T}} \frac{1}{\cos ^{2 m} \pi z} \pi N \cot (\pi N z) & d z \\
& =2 \sum_{k=1}^{\left\lfloor\frac{N-1}{2}\right\rfloor} \frac{1}{\cos ^{2 m} \frac{k \pi}{N}}+1+\operatorname{Res}_{z=\frac{1}{2}} \frac{1}{\cos ^{2 m} \pi z} \pi N \cot (\pi N z)
\end{aligned}
$$

by the residue theorem. From this we derive

$$
S_{2 m}(N)=\sum_{k=1}^{\left\lfloor\frac{N-1}{2}\right\rfloor} \frac{1}{\cos ^{2 m} \frac{k \pi}{N}}=-\frac{1}{2}-\frac{1}{2} \operatorname{Res}_{z=\frac{1}{2}} \frac{1}{\cos ^{2 m} \pi z} \pi N \cot (\pi N z) .
$$

In [4] the Bernoulli-Nörlund polynomials are introduced by the relation

$$
\frac{\omega_{1} \cdots \omega_{k} t^{k} e^{x t}}{\left(e^{\omega_{1} t}-1\right) \cdots\left(e^{\omega_{k} t}-1\right)}=\sum_{n=0}^{\infty} \frac{t^{n}}{n !} B_{n}^{(k)}\left(x ; \omega_{1}, \ldots, \omega_{k}\right) .
$$

We specialise $\omega_{1}=\cdots=\omega_{k}=2 i, x=k i$, and $t=\pi z$ to obtain

$$
\left(\frac{\pi z}{\sin \pi z}\right)^{k}=\sum_{n=0}^{\infty} \frac{(\pi z)^{n}}{n !} B_{n}^{(k)}(k i ; 2 i, \ldots, 2 i) .
$$

Writing $P_{n}^{(k)}=i^{n} B_{n}^{(k)}(k i ; 2 i, \ldots, 2 i)$ and observing that $P_{2 n+1}^{(k)}=0$ we have

$$
\frac{1}{\sin ^{k} \pi z}=\sum_{n=0}^{\infty} \frac{(\pi z)^{2 n-k}}{(2 n) !}(-1)^{n} P_{2 n}^{(k)}
$$

We have

$$
\operatorname{Res}_{z=\frac{1}{2}} \frac{1}{\cos ^{2 m} \pi z} \pi N \cot (\pi N z)=\operatorname{Res}_{z=0} \frac{1}{\sin ^{2 m} \pi z} \pi N \cot \left(\pi N z+\frac{N}{2} \pi\right) .
$$

Notice that

$$
\cot \left(\pi N z+\frac{N}{2} \pi\right)= \begin{cases}\cot (\pi N z) & \text { if } N \text { is even, } \\ -\tan (\pi N z) & \text { if } N \text { is odd. }\end{cases}
$$

Thus it is natural to distinguish two cases according to the parity of $N$.

From [3] we have

$$
\begin{aligned}
\pi \cot \pi z & =\sum_{n=0}^{\infty} \frac{\pi^{2 n} z^{2 n-1}}{(2 n) !}(-1)^{n} 4^{n} B_{2 n}, \\
\pi \tan \pi z & =\sum_{n=1}^{\infty} \frac{\pi^{2 n} z^{2 n-1}}{(2 n) !}(-1)^{n-1} 4^{n}\left(4^{n}-1\right) B_{2 n} .
\end{aligned}
$$


Then for even $N$ we have

$$
\begin{aligned}
& \operatorname{Res}_{z=\frac{1}{2}} \frac{1}{\cos ^{2 m} \pi z} \pi N \cot (\pi N z) \\
&=\left[z^{-1}\right] \sum_{\ell=0}^{\infty} \frac{(\pi z)^{2 \ell-2 m}}{(2 \ell) !}(-1)^{\ell} P_{2 \ell}^{(2 m)} \pi N \sum_{n=0}^{\infty} \frac{(N \pi z)^{2 n-1}}{(2 n) !}(-1)^{n} 4^{n} B_{2 n} \\
&=\frac{(-1)^{m}}{(2 m) !} \sum_{n=0}^{m}\left(\begin{array}{c}
2 m \\
2 n
\end{array}\right) P_{2(m-n)}^{(2 m)} B_{2 n}(2 N)^{2 n}
\end{aligned}
$$

and for odd $N$

$$
\begin{aligned}
& \operatorname{Res}_{z=\frac{1}{2}} \frac{1}{\cos ^{2 m} \pi z} \pi N \cot (\pi N z) \\
&=-\left[z^{-1}\right] \sum_{\ell=0}^{\infty} \frac{(\pi z)^{2 \ell-2 m}}{(2 \ell) !}(-1)^{\ell} P_{2 \ell}^{(2 m)} \pi N \sum_{n=1}^{\infty} \frac{(\pi N z)^{2 n-1}}{(2 n) !}(-1)^{n-1} 4^{n}\left(4^{n}-1\right) B_{2 n} \\
&=\frac{(-1)^{m}}{(2 m) !} \sum_{n=1}^{m}\left(\begin{array}{c}
2 m \\
2 n
\end{array}\right) P_{2(m-n)}^{(2 m)} B_{2 n}\left(4^{n}-1\right)(2 N)^{2 n} .
\end{aligned}
$$

Summing up, we have for even $N$

$$
S_{2 m}(N)=-\frac{1}{2}+\frac{(-1)^{m-1}}{2(2 m) !} \sum_{n=0}^{m}\left(\begin{array}{c}
2 m \\
2 n
\end{array}\right) P_{2(m-n)}^{(2 m)} B_{2 n}(2 N)^{2 n}
$$

and for odd $N$

$$
S_{2 m}(N)=-\frac{1}{2}+\frac{(-1)^{m-1}}{2(2 m) !} \sum_{n=0}^{m}\left(\begin{array}{c}
2 m \\
2 n
\end{array}\right) P_{2(m-n)}^{(2 m)} B_{2 n}\left(4^{n}-1\right)(2 N)^{2 n} .
$$

Equation (2) gives us for even $N$ :

$$
\begin{array}{rlrl}
m=1: & & \frac{1}{6} N^{2}-\frac{2}{3} \\
m=2: & \frac{1}{90} N^{4}+\frac{1}{9} N^{2}-\frac{28}{45} \\
m=3: & \frac{1}{945} N^{6}+\frac{1}{90} N^{4}+\frac{4}{45} N^{2}-\frac{568}{945} \\
m=4: & \frac{1}{9450} N^{8}+\frac{4}{2835} N^{6}+\frac{7}{675} N^{4}+\frac{8}{105} N^{2}-\frac{8336}{14175} \\
m=5: & \frac{1}{93555} N^{10}+\frac{1}{5670} N^{8}+\frac{13}{8505} N^{6}+\frac{82}{8505} N^{4}+\frac{64}{945} N^{2}-\frac{54176}{93555} \\
m=6: & \frac{691}{638512875} N^{12}+\frac{2}{93555} N^{10}+\frac{31}{141750} N^{8}+\frac{278}{178605} N^{6}+\frac{1916}{212625} N^{4}+\frac{128}{2079} N^{2}-\frac{365470016}{638512875} \\
m=7: & \frac{2}{18243225} N^{14}+\frac{691}{273648375} N^{12}+\frac{2}{66825} N^{10}+\frac{311}{1275750} N^{8}+\frac{592}{382725} N^{6}+\frac{944}{111375} N^{4} \\
m=8: & \quad \frac{3617}{325641566250} N^{16}+\frac{16}{547729675} N^{14}+\frac{113324}{28733079375} N^{12}+\frac{1072}{29469825} N^{10}+\frac{2473}{9568125} N^{8} \\
& \quad+\frac{134432}{88409475} N^{6}+\frac{8533792}{1064188125} N^{4}+\frac{1024}{19305} N^{2}-\frac{274946646272}{488462349375}
\end{array}
$$


Equation (2) gives us for odd $N$ :

$$
\begin{array}{rlrl}
m=1: & & \frac{1}{2} N^{2}-\frac{1}{2} \\
m=2: & & \frac{1}{6} N^{4}+\frac{1}{3} N^{2}-\frac{1}{2} \\
m=3: & & \frac{1}{15} N^{6}+\frac{1}{6} N^{4}+\frac{4}{15} N^{2}-\frac{1}{2} \\
m=4: & & \frac{17}{630} N^{8}+\frac{4}{45} N^{6}+\frac{7}{45} N^{4}+\frac{8}{35} N^{2}-\frac{1}{2} \\
m=5: & \frac{31}{2835} N^{10}+\frac{17}{378} N^{8}+\frac{13}{135} N^{6}+\frac{82}{567} N^{4}+\frac{64}{315} N^{2}-\frac{1}{2} \\
m=6: & \frac{691}{155925} N^{12}+\frac{62}{2835} N^{10}+\frac{527}{9450} N^{8}+\frac{278}{2835} N^{6}+\frac{1916}{14175} N^{4}+\frac{128}{693} N^{2}-\frac{1}{2} \\
m=7: & \frac{10922}{6081075} N^{14}+\frac{691}{66825} N^{12}+\frac{62}{2025} N^{10}+\frac{5287}{85050} N^{8}+\frac{592}{6075} N^{6}+\frac{944}{7425} N^{4}+\frac{512}{3003} N^{2}-\frac{1}{2} \\
m=8: & \frac{929569}{1277025750} N^{16}+\frac{87376}{18243225} N^{14}+\frac{113324}{7016625} N^{12}+\frac{33232}{893025} N^{10}+\frac{42041}{637875} N^{8} \\
& \quad+\frac{134432}{1403325} N^{6}+\frac{8533792}{70945875} N^{4}+\frac{1024}{6435} N^{2}-\frac{1}{2}
\end{array}
$$

For the cosecant sum, we start with the contour integral

$$
\frac{1}{2 \pi i} \oint_{R_{T}} \frac{1}{\sin ^{2 m} \pi z} \pi N \cot (\pi N z) d z
$$

which is again zero and, by summing residues, leads to the equation

$$
0=\sum_{k=1}^{\left\lfloor\frac{N-1}{2}\right\rfloor} \frac{1}{\sin ^{2 m} \frac{k \pi}{N}}+\frac{1}{2} \operatorname{Res}_{z=0} \frac{1}{\sin ^{2 m} \pi z} \pi N \cot (\pi N z)+\frac{1+(-1)^{N}}{4} .
$$

We observe that

$$
\sum_{k=1}^{\left\lfloor\frac{N-1}{2}\right\rfloor} \frac{1}{\sin ^{2 m} \frac{k \pi}{N}}+\frac{1+(-1)^{N}}{4}
$$

equals the residue that we already calculated for $S_{2 m}(N)$ and $N$ even. Thus we have

$$
C_{2 m}(N)=\sum_{k=1}^{\left\lfloor\frac{N-1}{2}\right\rfloor} \frac{1}{\sin ^{2 m} \frac{k \pi}{N}}=\frac{(-1)^{m}}{(2 m) !} \sum_{n=0}^{m}\left(\begin{array}{c}
2 m \\
2 n
\end{array}\right) P_{2(m-n)}^{(2 m)} B_{2 n}(2 N)^{2 n}-\frac{1+(-1)^{N}}{4} .
$$

\section{Computing $P_{2 n}^{(2 m)}$}

In this section we want to have a closer look at the Laurent series expansion of $\sin ^{-2 m} \pi z$. Our approach is somewhat similar to the one used in [1].

We start with the expansion (5). Differentiating yields

$$
\frac{1}{\sin ^{2} \pi z}=\sum_{n=0}^{\infty} \frac{(\pi z)^{2 n-2}}{(2 n) !}(2 n-1)(-1)^{n-1} 4^{n} B_{2 n} .
$$

This gives

$$
P_{2 n}^{(2)}=-(2 n-1) 4^{n} B_{2 n}
$$


Differentiating $\sin ^{-2 m} \pi z$ twice yields

$$
\frac{d^{2}}{d z^{2}} \frac{1}{\sin ^{2 m} \pi z}=2 m(2 m+1) \pi^{2} \frac{1}{\sin ^{2 m+2} \pi z}-4 m^{2} \pi^{2} \frac{1}{\sin ^{2 m} \pi z} .
$$

We now write

$$
\frac{1}{\sin ^{2 m} \pi z}=H_{2 m}(z)+R_{2 m}(z)=\frac{1}{(2 m-1) !} \sum_{\ell=1}^{m} \frac{(2 \ell-1) ! b_{2 \ell}^{(2 m)} 4^{m-\ell}}{(\pi z)^{2 \ell}}+R_{2 m}(z),
$$

where $H_{2 m}$ is the principal part around $z=0$ and $R_{2 m}$ denotes the regular part. Since differentiation preserves principal and regular parts, (11) gives

$$
H_{2 m}^{\prime \prime}(z)=\pi^{2} 2 m(2 m+1) H_{2 m+2}(z)-4 m^{2} \pi^{2} H_{2 m}(z),
$$

which gives the recursion (setting $b_{0}^{(2 m)}=b_{2 m+2}^{(2 m)}=0$ and $b_{2}^{(2)}=1$ )

$$
b_{2 \ell}^{(2 m+2)}=m^{2} b_{2 \ell}^{(2 m)}+b_{2 \ell-2}^{(2 m)} \text { for } 1 \leq \ell \leq m+1 .
$$

This recursion shows that the numbers $b_{2 \ell}^{(2 m)}$ are given by

$$
\sum_{\ell=0}^{m} b_{2 \ell}^{(2 m)} x^{2 \ell}=\prod_{k=0}^{m-1}\left(x^{2}+k^{2}\right)
$$

Thus they are closely related to the central factorial numbers $t(n, k)$ studied in [5, p. 213]:

$$
x \prod_{k=1}^{m-1}\left(x^{2}-k^{2}\right)=\sum_{k=0}^{2 m} t(2 m, 2 k+1) x^{2 k+1}
$$

and a similar expression for odd first argument. This gives $b_{2 \ell}^{(2 m)}=(-1)^{\ell+m} t(2 m, 2 \ell)$. We notice that the polynomials in (15) appear mutatis mutandis in [2] as differential operators. These operators are used to model the recursion (13).

In Table 1 we computed the values $b_{2 k}^{(2 m)}$ for small values of $m$.

\begin{tabular}{r||r|r|r|r|r|r|r|r|r|}
$b_{k}^{(m)}$ & $k=2$ & 4 & 6 & 8 & 10 & 12 & 14 & 16 & 18 \\
\hline$m=2$ & 1 & & & & & & & & \\
4 & 1 & 1 & & & & & & & \\
6 & 4 & 5 & 1 & & & & & & \\
8 & 36 & 49 & 14 & 1 & & & & & \\
10 & 576 & 820 & 273 & 30 & 1 & & & & \\
12 & 14400 & 21076 & 7645 & 1023 & 55 & 1 & & & \\
14 & 518400 & 773136 & 296296 & 44473 & 3003 & 91 & 1 & & \\
16 & 25401600 & 38402064 & 15291640 & 2475473 & 191620 & 7462 & 140 & 1 & \\
18 & 1625702400 & 2483133696 & 1017067024 & 173721912 & 14739153 & 669188 & 16422 & 204 & 1 \\
\hline
\end{tabular}

TABLE 1. Table of $b_{k}^{(m)}$ for small values of $m$ (compare with [5, Table 6.1, p. 217]) 
We now consider the Mittag-Leffler expansion

$$
\frac{1}{\sin ^{2 m} \pi z}=\sum_{n \in \mathbb{Z}} H_{2 m}(z+n)=H_{2 m}(z)+\sum_{n=1}^{\infty}\left(H_{2 m}(z+n)+H_{2 m}(z-n)\right) .
$$

Expanding the last sum into a power series and using (12) yields

$$
\frac{1}{\sin ^{2 m} \pi z}=H_{2 m}(z)+\frac{4^{m}}{(2 m-1) !} \sum_{k=0}^{\infty} \frac{(\pi z)^{2 k}}{(2 k) !} 4^{k}(-1)^{k} \sum_{\ell=1}^{m}(-1)^{\ell-1} \frac{1}{2 \ell+2 k} b_{2 \ell}^{(2 m)} B_{2 \ell+2 k},
$$

where we have used $\zeta(2 k)=(-1)^{k-1} \frac{2^{2 k-1} \pi^{2 k}}{(2 k) !} B_{2 k}$. This gives

$$
P_{2 k}^{(2 m)}= \begin{cases}2 m\left(\begin{array}{c}
2 k \\
2 m
\end{array}\right) 4^{k} \sum_{\ell=0}^{m-1}(-1)^{\ell-1} \frac{1}{2 k-2 \ell} b_{2 m-2 \ell}^{(2 m)} B_{2 k-2 \ell} & \text { for } k \geq m, \\
(-1)^{k} 4^{k} b_{2 m-2 k}^{(2 m)} /\left(\begin{array}{c}
2 m-1 \\
2 k
\end{array}\right) & \text { for } 0 \leq k \leq m-1 .\end{cases}
$$

Inserting this into (6) and (7) yields for even $N$

$$
S_{2 m}(N)=\frac{4^{m-1}}{(2 m-1) !} \sum_{\ell=1}^{m} \frac{(-1)^{\ell-1}}{\ell} b_{2 \ell}^{(2 m)} B_{2 \ell} N^{2 \ell}-\frac{4^{m-1}}{(2 m-1) !} \sum_{\ell=1}^{m} \frac{(-1)^{\ell-1}}{\ell} b_{2 \ell}^{(2 m)} B_{2 \ell}-\frac{1}{2}
$$

and for odd $N$

$$
S_{2 m}(N)=\frac{4^{m-1}}{(2 m-1) !} \sum_{\ell=1}^{m} \frac{(-1)^{\ell-1}}{\ell} b_{2 \ell}^{(2 m)} B_{2 \ell}\left(4^{\ell}-1\right) N^{2 \ell}-\frac{1}{2}
$$

Similarly, we obtain

$$
\begin{aligned}
C_{2 m}(N)=\frac{4^{m-1}}{(2 m-1) !} \sum_{\ell=1}^{m} \frac{(-1)^{\ell-1}}{\ell} b_{2 \ell}^{(2 m)} & B_{2 \ell} N^{2 \ell} \\
& \quad-\frac{4^{m-1}}{(2 m-1) !} \sum_{\ell=1}^{m} \frac{(-1)^{\ell-1}}{\ell} b_{2 \ell}^{(2 m)} B_{2 \ell}-\frac{1+(-1)^{N}}{4} .
\end{aligned}
$$

\section{REFERENCES}

[1] K Dilcher, Sums of products of Bernoulli numbers, J. Number Theory 60 (1996), 23-41.

[2] N. Gauthier and P. S. Bruckman, Sums of even integral powers of the cosecant and the secant, Fibonacci Quart. 44 (2007), 264-273.

[3] R.L. Graham, D.E. Knuth, and O. Patashnik, Concrete mathematics: A foundation for computer science, second ed., Addison Wesley, Reading, MA, 1994.

[4] N. E. Nörlund, Differenzenrechnung, Grundlehren der mathematischen Wissenschaften, vol. XIII, Springer Verlag, Berlin, 1924.

[5] J. Riordan, Combinatorial identities, John Wiley \& Sons Inc., New York, 1968. 
(P. Grabner) Institut für Mathematik A, Technische Universität Graz, Steyrergasse 30, 8010 Graz, Austria

E-mail address: peter.grabner@tugraz.at

(H. Prodinger) Department of Mathematics, University of Stellenbosch, 7602 StellenBosch, South Africa

E-mail address: hproding@sun.ac.za 